\title{
Effect of polyvalent bacterial lysate on the clinical course of pollen allergic rhinitis in children
}

\author{
Kamil P. Janeczek ${ }^{1}$, Andrzej Emeryk ${ }^{1}$, Piotr Rapiejko² \\ ${ }^{1}$ Department of Pulmonary Diseases and Children Rheumatology, Medical University of Lublin, Lublin, Poland \\ 2Department of Otolaryngology with Division of Cranio-Maxillo-Facial Surgery in Military Institute of Medicine, Warsaw, Poland \\ Adv Dermatol Allergol 2019; XXXVI (4): 504-505 \\ DOI: https://doi.org/10.5114/ada.2019.87457
}

Seasonal allergic rhinitis (SAR) is caused by various allergens, including wind-pollinated plants, and mainly by grass pollen allergens in Poland. The disease reduces the quality of life of patients and requires intensive treatment in the pollen season [1].

Polyvalent bacterial lysates (PBL) have been used for many years to prevent respiratory tract infections in children and adults [2]. Recent research also draws attention to the immunoregulatory potential of PBL, indicating the possibility of their future use in the prevention and treatment of atopic dermatitis, AR and asthma [3-7].

The aim of the study was to assess the impact of PBL on the clinical course of childhood SAR.

The study included 38 children aged 5-17 years (27 males; mean age: $9 \pm 3.2$ years) with SAR caused by grass pollen, randomly selected from the group of children treated at the Outpatient Clinic of the University Children's Hospital in Lublin. Main inclusion criteria were: SAR symptoms for at least two last grass pollen seasons, positive skin prick test results with grass pollen allergens and/or serum allergen specific immunoglobulin E (IgE) level $>0.70 \mathrm{lU} / \mathrm{ml}$. The main exclusion criteria were: allergen immunotherapy over the last 3 years, presence of other chronic diseases of the nose and/or paranasal sinuses.

All children underwent standard SAR treatment from the beginning of the grass pollen season (oral antihistamines or/and intranasal corticosteroids "on demand"). Patients were randomly assigned to two groups. One group $(n=20)$ received mechanical PBL (PMBL-Ismigen ${ }^{\circledR}$, Lallemand Pharma AG, Switzerland) (group P) during the grass pollen season, sublingually for 10 consecutive days per month for 3 consecutive months (May, June and July 2017). The pollen season in Lublin started on 19 May 2017 and ended on 12 August 2017. The remaining patients (control group - group C, $n=18$ ) did not receive PMBL. Throughout the study, the children's parents filled in the patient's diary every day, when the symptoms of SAR were noticed. The study included two 5 -week periods during which patients had 3 visits (V): V1 - screening/randomization visit (19.05-30.05.2017), V2 (23.06-5.07.2017) and V3 (1.08-12.08.2017).

The severity of SAR symptoms and associated eye symptoms were assessed by the Total Nasal Symptom Score (TNSS) and Total Ocular Symptom Score (TOSS). Nasal Peak Inspiratory Flow (PNIF) values were measured by Youlten Peak Flow Meter (Clement Clark International, UK). Nasal symptoms (obstruction, sneezing, rhinorrhea, itching) and eye symptoms (red, itchy, watery eyes) were scored using a four-point scale ( 0 = no symptom; $1=$ mild; 2 = moderate; 3 = severe) and the sum was calculated as TNSS and TOSS, respectively. The average values of TNSS and TOSS obtained at the beginning (from the period of 7 days after V1 - T0), at the peak (from the period of 7 days before $V 2-T 1$ ) and at the end of the grass pollen season (from the period of 7 days before $\mathrm{V} 3-\mathrm{T} 2$ ) and PNIF values from each visit were used for statistical analysis (ANOVA test, Mann-Whitney U-test).

Study was approved by the Bioethics Committee of the Medical University in Lublin (no. KE-0254/41/2017). Patients had to give written informed consent to participate in the study.

All children from group $P$ and 16 children from group C completed the study and treatment was well tolerated. In patients treated with PMBL, a statistically significant reduction in the symptoms of SAR as compared to the group without PMBL at the end of the grass pollen season was observed. TNSS significantly increased in group $C$ $(\mathrm{TO}=2.84 \pm 2.72, \mathrm{~T} 2=4.77 \pm 2.74)(p=0.009)$; consequently significantly decreased in group $\mathrm{P}(\mathrm{TO}=3.33$ $\pm 2.20 ; \mathrm{T} 2=1.79 \pm 1.57)(p=0.001)$, as reported in Figure 1. The intergroup comparison was the difference at T2 $(p=$ $0.001)$. PNIF value increased in group $C$ between TO and $\mathrm{T} 2(\mathrm{TO}=75.00 \pm 29.81, \mathrm{~T} 2=79.29 \pm 46.32)(p=0.724)$ and

Address for correspondence: Kamil P. Janeczek MD, PhD, Department of Pulmonary Diseases and Children Rheumatology, Medical University of Lublin, 6 Prof. Antoniego Gębali St, 20-093 Lublin, Poland, phone: +48 81718 54 53, e-mail: kamil.janeczek@umlub.pl Received: 16.08.2018, accepted: 22.08.2018. 
significantly increased in group P (TO = $79.55 \pm 30.78$; T2 $=108.86 \pm 41.69)(p=0.009)$. The intergroup comparison showed a significant difference at T2 $(p=0.021)$. The decrease in TOSS values in group P did not show statistical significance.

Nasal corticosteroids and oral antihistamines use were overlapping: 24 days in group $C$ and 22 days in group $P$, and 20 days in group $C$ and 18 days in group $P$, respectively.

The study demonstrated that PMBL was able to significantly improve SAR symptoms in children. The results require confirmation; in the next grass pollen season (2018), a randomized, double-blind, placebo-controlled trial will be conducted.

\section{Conflict of interest}

The authors declare no conflict of interest.

\section{References}

1. Devillier P, Bousquet J, Salvator $\mathrm{H}$, et al. In allergic rhinitis, work, classroom and activity impairments are weakly related to other outcome measures. Clin Exp Allergy 2016; 46: 1456-64.

2. Cazzola M, Anapurapu S, Page CP, et al. Polyvalent mechanical bacterial lysate for the prevention of recurrent respiratory infections: a meta-analysis. Pulm Pharmacol Ther 2012; 25: 62-8.

3. Koatz AM, Coe NA, Cicerán A, et al. Clinical and immunological benefits of OM- 85 bacterial lysate in patients with allergic rhinitis, asthma, and COPD and recurrent respiratory infections. Lung 2016; 194: 687-97.

4. Lau S, Gerhold K, Zimmermann K, et al. Oral application of bacterial lysate in infancy decreases the risk of atopic dermatitis in children with 1 atopic parent in a randomized,

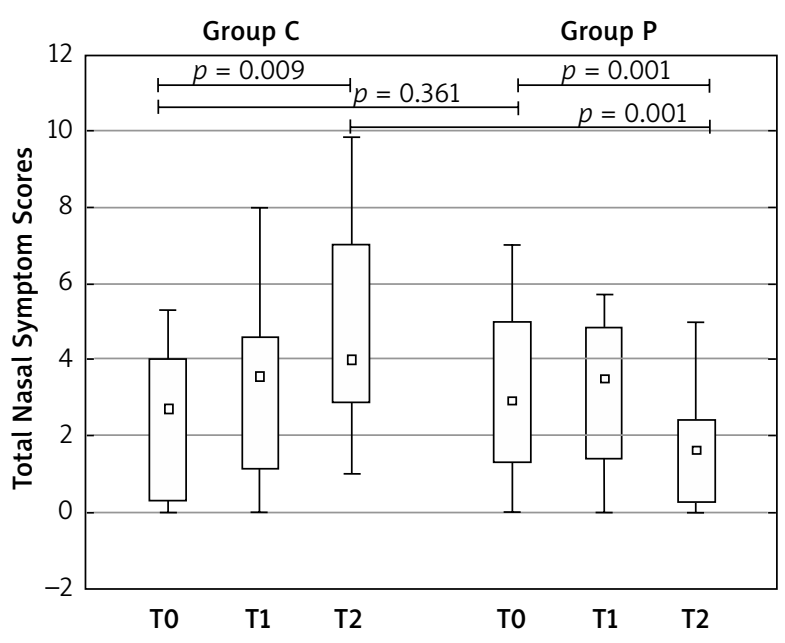

Figure 1. Total Nasal Symptom Scores in patients treated with PMBL (group P) or without PMBL (group C) at the beginning (TO), in the middle (T1), and at the end (T2) of the pollen season. Data are expressed as medians, interquartile ranges, upper and lower extreme

placebo-controlled trial. J Allergy Clin Immunol 2012; 129: 1040-7.

5. Razi CH, Harmanci K, Abaci A, et al. The immunostimulant OM-85 BV prevents wheezing attacks in preschool children. J Allergy Clin Immunol 2010; 126: 763-9.

6. Banche G, Allizond V, Mandras N, et al. Improvement of clinical response in allergic rhinitis patients treated with an oral immunostimulating bacterial lysate: in vivo immunological effects. Int J Immunopathol Pharmacol 2007; 20: 129-38.

7. Edwards MR, Walton RP, Jackson DJ, et al. The potential of anti-infectives and immunomodulators as therapies for asthma and asthma exacerbations. Allergy 2018; 73: 50-63. 